\title{
MICROHONGOS DE LA PATAGONIA CHILENA: ALGUNOS Ascomycetes COPROFILOS
}

\author{
(Microfungi of the Chilean Patagonia: Some coprophilous Ascomycetes)
}

\author{
Eduardo. Piontelli, L. \& Maria Angélica Grixolli, A. \\ Universidad de Valparaiso, Escuela de Medicina \\ Cátedra de Micologia, Casilla 92 V. Valparaíso, CHILE.
}

Palabras clave: Hongos coprófilos, Ascomyeetes, Patagonia chilena. Key words: Coprophilous fungi, Ascomycetes, chilean Patagonia

\section{RESUMEN}

Contimuando los estudios sobre hongos coprófilos, en el periodo estival de 1997 se obhwieron 21 muestras de excrementos de hovinos de la Patagonia chilena (Regián de Aisén), en un área geográfica comprendida enure Villa Mañiguales, Puerto Aisén, Coihaique y el lago Elizalde

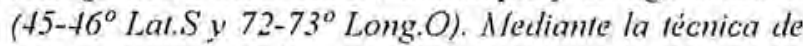
cullivo en cámara humeda e incubandose a temperanura ambiente, se detectó la presencia de un total de 12 géneros y 27 especies de Ascomycetes.

Los géneros con mavor cantidad de especies fueron: Podospora (7), Ascobolus (3) y Coprotus (3) y las especies dominantes: Podospora argentinensis (90,5\%), Ascobolus immersus $(85,7 \%), P$. miniglutinans $(71,-1 \%)$ e Iodophanus carneus (62\%).

Once especies se consideraron frecuentes, entre ellas: Podospora fimiseda ( $+7,6 \%)$, Sporormiella minima (12,9\%), S. intermedia (33,3\%), Saccobolus depauperatus $(33,3 \%)$, Coniochueta leucoplaca (23,9\%), Coprotus granuliformis $(23,9 \%)$.

De las especies consideradas esporádicas, destacan por ser sus primeros aislamientos en Chile: Arnium leporinum, Podospora conica, Pyxidiophora badiorostris, P. grovei, Thecotheus holmskjoldii, T.pelletieri y Coprotus niveus.

Se compararon las dimensiones de las ascosporas de todas las cepas estudiadas con los datos registrados en la literanua en ambos hemisferios, comprobandose que algunos especies de Podospora (principalmente), Ascobolus y Thecotheus, presentan ecotipos que difieren levemente (largo y ancho).

\section{SUMMARY}

To study even further the coprophitois fungi in the summer period of 1997, 21 samples of caule faeces from the chilean Patagonia (Región of Aisén), in a geographical area involving from Villa Mañiguales, Puerto Aisén, Coihaique and lake Elizalde $\left(45-46^{\circ}\right.$ S.Lat. and $72-73^{\circ} \mathrm{W}$ Long.) were collected. By means of the culture in wet chamber technique and the incubation at room temperature, the presence of a total 12 genera and 27 species of Ascomycetes was detected.

Genera exhibing greatest number of species were: Podospora (7), Ascobolus (3) and Coprotus (3) and dominant species: Podospora argentinensis (90,5\%), Ascobolus immersus $(85,7 \%), P$. miniglutinans $(71,4 \%)$ and Iodophanus carneus (62\%).

Eleven species were considered as frecuent, among those: Podospora fimiseda (47,6\%), Sporormiella minima (42,9\%), S. intermedia (33,3\%), Saccobolus depauperatus $(33,3 \%)$, Coniochaeta leucoplaca $(23,9 \%)$, Coprotus granuliformis (23,9\%).

Among those species considered as sporadic, the following are remarkable for having their first isolation in Chile: Arnium leporinum, Podospora conica, Pyxidiophora badiorostris, P. grovei, Thecoteus holmskjoldii, T.pelletieri,and Coprotus niveus.

Dimension of the ascospores of every strain studied were compared to data recorded in literature in both hemisphere and it was ascertained that some species of Polospora(mainly) Ascobolus and Thecotheus, exhibit ecotype differing slighly(in leng and width). 


\section{INTRODUCCION}

$\mathrm{El}$ estudio de los hongos coprófilos ofrece interesantes aspectos biológicos, ecológicos y taxonómicos, mientras su diversidad y mecanismos de competencia, ofrecen actualmente interesantes expectativas en la producción de variadas sustancias antagónicas útiles en diversos campos de las ciencias (Bills, 1995; Milier, 1995; Shearer, 1995).

Los estudios sobre hongos coprófilos realizados a nivel nacional son escasos, limitandose a localidades del centroy sur del pais (Spegazzini 1921; Lazo, 1979, Udagawa, 1980; Piontelli et al.,1981; Muroi \& Udagawa, 1984; Valldosera \& Guarro, 1988).

Las zonas vegetacionales higromórficas de la Patagonia chílena con sus particulares condiciones climáticas y edáficas, son aptas para la crianza de bovinos, situación de particular interés, para el estudio de sus poblaciones de microhongos coprófilos.

El presente trabajo tiene como objetivo aportar nuevos datos ecológicos sobre presencia y distribución de algunos taxa de Ascomycetes en ésta zona geográfíca.

\section{MATERIALESYMETODOS}

\section{Areade Muestreo}

En el período estival de 1997, se obtuvieron 21 muestras de excrementos bovinos (la mayoria en condiciones secas) de la Patagonia chilena (Región de Aisén), en un área geográfica comprendida entre Villa Mañiguales, Puerto Aisén, Coihaique y el Lago Elizalde ( $45-46^{\circ}$ Lat.S y $72-73^{\circ}$ Long.O) (Ver área geográfica).

\section{Perfil Biogeográfico}

Presenta un relieve diferente al resto del pais, la cordillera de la costa desaparece bajo el mar, mientras el Océano Pacífico penetra hacia el interior de la región, pudiendo llegar en algunos casos hasta la base de la Cordillera de los Andes (Cordillera de la Patagonia). Suclima es maritimo lluvioso y su actividad egropecuaria es escasa debido al clima adverso.

La vegetación en los suelos planos, es apta para el pastoreo y esta compuesta principalmente por Juncaceae y Cyperaceae (Juncus spp.,Carex spp.), pero también por varias gramíneas palatables para el ganado (especies de Agrostis, Bromus, Deschampia, Festuca, Hordeum, Elymus, Poa, Holcus, entre otras (Montaldo 1976). Sus bosques mixtos contienen generalmente coigue ( $\mathrm{No}$ thofagus betuloides), mañio (Saxegotea conspicua), canelo (Drimys winteri), lenga (Nothofagus pumilio), entre otros (Quintanilla, 1983).

\section{Método}

Se usó la técnica de cultivo en cámara húmeda, colocando los excrementos en 21 placas de Petri de $10 \mathrm{~cm}$, adicionando 5 a $10 \mathrm{ml}$ de agua destilada estéril, e incubando

\section{Area geográfica de muestreo}

REGIÓN AISÉN DEL GENERAL CARLOS IBÁNíZ DEL CAMPO

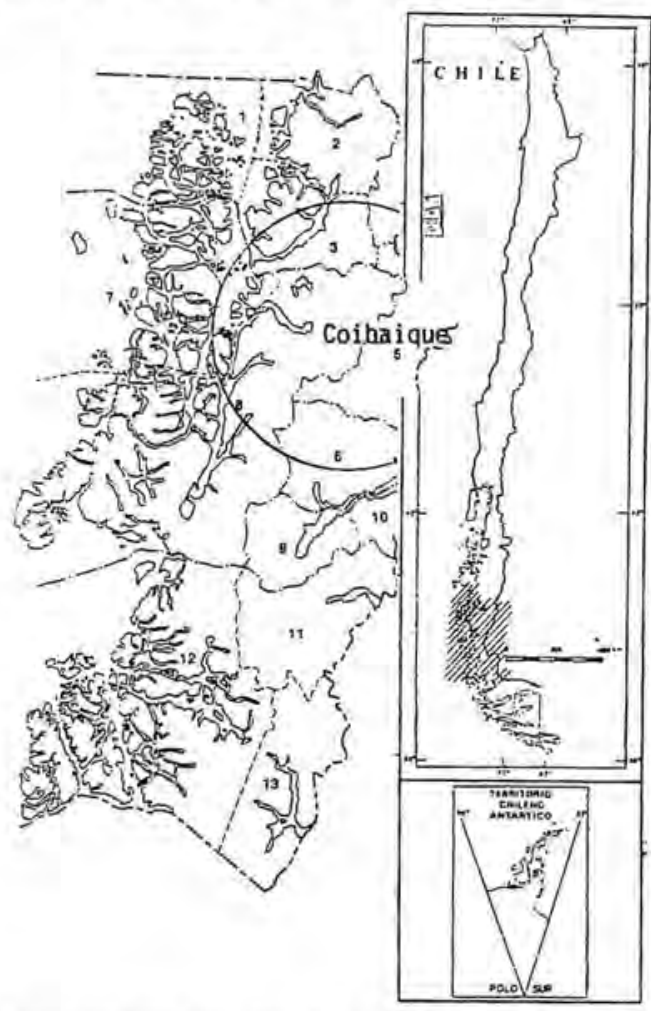

el material a temperatura ambiente $\left(20-24^{\circ} \mathrm{C}\right)$, por un periodo no mayor de 40 dias. Para mantener la humedad en el tiempo de incubación, se adicionó agua estéril a las placas cada 10 dias.

Las observaciones macroscópicas se efectuaron bajo lupa estereoscópica cada 10 días. Las microscópicas, para la determinación de los taxa, mediante preparaciones con lactofenol solo o lactofenol y azul de algodón.

Algunos aislamientos fue posible obtenerlos en agar excremento, preparado con el mismo sustrato $(100 \mathrm{~g} / \mathrm{l})$.

Cada especie fue contabilizada 1 sola vez en cada placa, no importando si su presencia era mayor. Los porcentajes de presencia de las diferentes categorías ecológicas consideradas (Dominantes, Frecuentes y Esporádicas), se efectuaron de la siguiente manera:

a) La presencia de una especie en 11 placas o más, se consideró como Dominante.

b) La presencia de una especie en 3 a 10 placas, se consideró como Frecuente

c) La presencia de una especie en 1 o 2 placas, se consideró como Esporádica. 
Las ascosporas de los taxa aislados se midieron con aumentomáximo (1000X), seleccionandose al azar, unnúmero no menor de 20 esporas maduras por cepa.

\section{RESULTADOS}

Se aislaron un total de 12 géneros y 27 especies de Ascomycetes que se repartieron en 3 categorías ecológicas (Gráficol), donde solo 4 de 27 especies se consideraron dominantes: Ascobolus inmmersus, Iodophanus carneus, Podospora argentinensis y P, miniglutinans (Tabla.1).

Gráfico 1. Distribución de las especies en categorías ecológicas

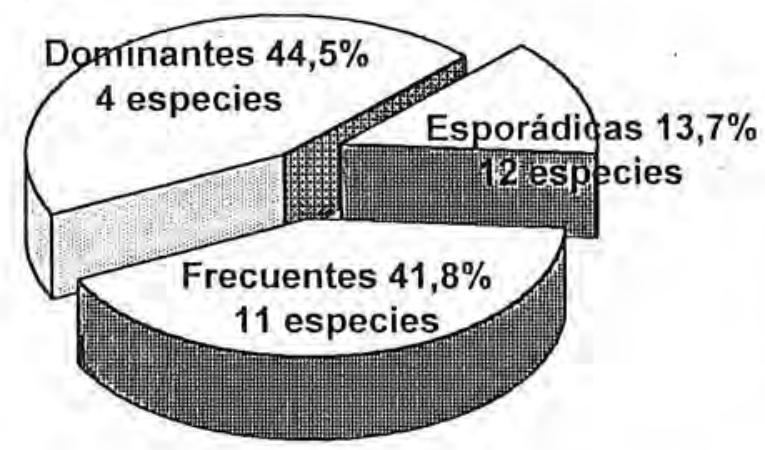

Gráfico 2. Géneros con mayor número de especies

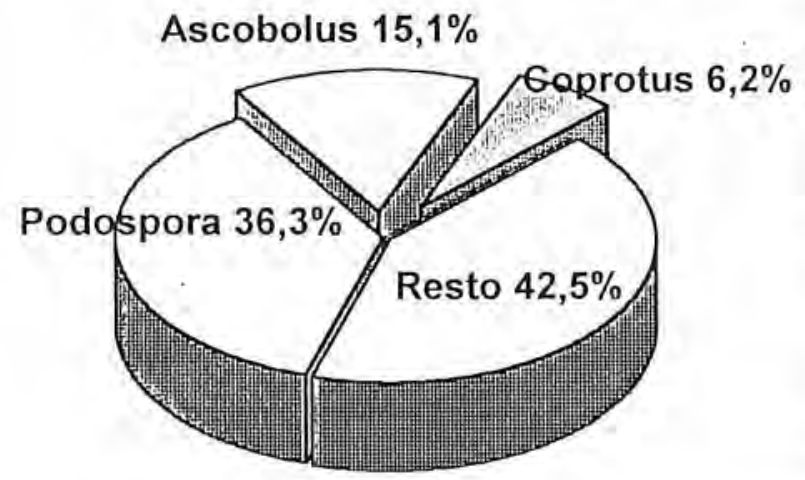

Las géneros con más spp. fueron Podospora (7), Ascobolus (3) y Coprotus (3) (Tabla 1, Gráfico. 2).

Podospora argentinensis, obtuvo la mayor presencia en el sustrato, mientras la totalidad de las especies integrantes del género, la más alta distribución en las muestras $(36,3 \%$ ) (Tabla 1, Gráfico . 2).

Las especies con mayor frecuencia fueron: Podospora fimiseda, Sporormiella minima, S. intermedia y Saccobolus depauperatus, mientras de las especies consideradas esporádicas: Arnium leporinum, Podospora conica, Pyxidiophora badiorostris, P. grovei, Thecotheus apiculatus, T.holmskjoldii y Coprotus niveus, destacan por ser sus primeros aislamientos en Chile.

Se compararon las dimensiones de las ascosporas de todas las cepas estudiadas con los datos registrados en la literatura en ambos hemisferios, comprobandose que algunas especies de Podospora (principalmente), Ascobolus, Pyxidiophora y Thecotheus, presentan ecotipos con ciertas diferencias, en largo y ancho, de los datos registrados en la literatura (Tabla .1, Gráfico 3).

\section{DISCUSION}

Nuestros resultados han permitido obtener un panorama regional de un conjunto de especies de Ascomycetes coprófilo de la Patagonia chilena, algunos de los cuales descritos anteriormente en el país en diferentes tipos de excrementos de herbivoros (Spegazzini, 1921;Lazo, 1969, 1979; Udagawa, 1980; Piontelliet.al 1981; Muroi \&Udagawa 1984). A pesar que muchos de estos hongos son cosmopolitas, especialmente los de bovinos, su distribución depende principalmente del tipo de animal, la ubicación geográfica, el clima y la naturaleza quimica del sustrato (Wicklowetal.,1980).

La Patagonia chilena, por su clima templado - frío y lluvioso, con un abundante perfil vegetacional, ofrece condiciones particulares para la crianza de bovinos y ovinos y por ende presenta un habitat favorable para la dispersión de especies geofilicas y coprófilas (Yocom \& Wicklow,1980)

Cabe destacar que nuestros aislamientos patagónicos, presentan algunas especies de Thelebolus, Sporormiella, Saceobolus, Lasiobolus, Coprotus y Iodophanus, que anteriormente fueron aisladas en equinos y otros herbivoros en la zona central ( Lazo 1979; Piontelli etal, 1981).

El género Podospora, representó cualitativa y cuantitativamente el porcentaje más alto de colonización del sustrato, siendo un buen ejemplo de distribución regional y uno de los géneros coprófilos más aislados en nuestro estudio. Muroi \& Udagawa (1984), aislaron en zonas más australes solo 2 especies de Podospora, coincidiendo solo una con nuestros aislamientos (P.pleiospora), mientras ninguna de las 6 especies aisladas de la zona central por Piontelli et al .(1981), fueron registradas en la zona patagófica. Estoparece confirmar que solo algunos representantes del taxon tienen amplia distribución, independientemente del sustrato y el clima (P.miniglutinans, P.fimiseda, P.pleiospora, P.conica), mientras otros, pueden mantener una relación más estrecha con estas variables (P.argentinensis, P.ostlingospora).

$P$. argentinensis, la especie dominante del género, tiene al parecer en la literatura una distribución restringida a la Argentina (Spegazzini,1912), Mexico y USA (Mirza \& 
Tabla 1. Ascomycetes coprófílos de la Patagonia chilena (21 muestras)

\begin{tabular}{|c|c|c|c|c|}
\hline \multirow{2}{*}{ Taxa $n=27$} & \multirow[b]{2}{*}{$\%$} & \multirow[b]{2}{*}{ Categ. } & \multicolumn{2}{|c|}{ Ascosporas* } \\
\hline & & & $x(\mu \mathrm{m})$ & Rango $(\mu \mathrm{m})$ \\
\hline Arnium leporinum (Cain)Lundq.\& Krug & 9,5 & Esp. & $22,2 \times 13$ & $21,3-23,3 \times 12,613,6$ \\
\hline Ascobolus immersus Pers. per Pers. & 85,7 & Dom. & $57,3 \times 31,3$ & $50,4-68 \times 29,1-35,9$ \\
\hline A. perplexans Massee \& Salmon & 14,3 & Frec. & $21,5 \times 11,3$ & $19,4-24,3 \times 9,7-12,6$ \\
\hline A. furfuraceus Pers, ex Hook & 4,8 & Esp. & $23,7 \times 12,5$ & $22,3-25,2 \times 10,7-13$ \\
\hline Coniochaeta leucoplaca (Berk \& Rav.)Cain & 23,9 & Frec. & $7,8 \times 6,1$ & $6,8-7,8 \times 5,8-6,8$ \\
\hline Coprotus duplus Kimbrogh et al. & 14,3 & Frec. & $10,1 \times 5,8$ & $9,2-10,8 \times 5,3,6,8$ \\
\hline C. granuliformis (Cr.\&.Cr.) Kimbrough & 23,9 & Frec. & $11,2 \times 7,1$ & $9,7-11,7 \times 5.8-7,8$ \\
\hline C. niveus (Fuckel) Kimbrough et al. & 4,8 & Esp. & $9,5 \times \quad 5,5$ & $x 5-7$ \\
\hline Iodophanus carneus (Pers. ex Pers.) Korf & 62 & Dom. & $19,5 \times 11,8$ & $18,4-21,4 \times 11,6-12,6$ \\
\hline Lasiobolus intermedius Bezerra \& Kimbr. & 23,9 & $\overline{\text { Frec. }}$ & $18,7 \times 11,5$ & $17,5-19,4 \times 9,2-11,7$ \\
\hline Podospora argentinensis (Speg) Mirza \& Cain & 90,5 & Dom. & $33,9 \times 18,4$ & $29,1-37,8 \times 16,5-20,4$ \\
\hline P.conica (Fchl.) Bell \& Mahoney & 9,5 & $\overline{\text { Esp. }}$ & $24 \times 14$ & $23,3-25,2 \times 13,6-14,6$ \\
\hline P. fimiseda (Ces.\& DeNot.)Niessl(=P.fimicola) & 47,6 & Frec. & $55,8 \times 28,6$ & $55,3-58,2 \times 25,5-29,1$ \\
\hline P. miniglutinans Mirza \& Cain & 71,4 & Dom. & $24 \times 13,3$ & $20,-4-25,5 \times 12,6-13,6$ \\
\hline P.ostlingospora Cain & 14,3 & Frec. & $56,2 \times 29,3$ & $55,3-61,1 \times 27,2-30,1$ \\
\hline P. pleiospora (Winter) Niessl & 9,5 & Esp. & $31 \times 17,4$ & $29,1-34 \times 16,5-18,5$ \\
\hline Podospora sp. & 9,5 & Esp. & $19,2 \times 11,6$ & $17,5-20,4 \times 10,8-12,3$ \\
\hline Pyxidiophora badiorostris Lundqvist & 9,5 & Esp. & $43,3 \times 4,9$ & $42,7-48,5 \times 4,5-5,5$ \\
\hline P. grovei (Hawksw. \& Webst.) Lundqvist & 9,5 & Esp. & $4+, 2 \times 4,1$ & $39-53,5 \times 3,9-4,9$ \\
\hline Saccobolus citrinus Boud. \& Torrend & 9,5 & Esp. & $19,1 \times 9,7$ & $18,5-20,4 \times 8,8-9,7$ \\
\hline S. depauperatus (Berk.\& Broome) E.C.Hansen & 33,3 & Frec. & $13,7 \times 7,5$ & $11,7-14,6 \times 5,8-6,8$ \\
\hline Sporormiella intermedia (Auersw.)Ahmed \& Cain & 33,3 & Frec. & $50 \times 10,3$ & $48,5-54.4 \times 8,8-11,9$ \\
\hline S. minima Auersw. Ahmed \& Cain & 42,9 & Frec. & $28,5 \times 5,1$ & $27,2-31 \times 4,9-5,2$ \\
\hline Thecotheus holmskjoldii (Hansen) Eckblad & 4,8 & Esp. & 29) $\times 14,5$ & $28,8-31 \times 1+-15$ \\
\hline T. pelletieri (Cr.\& Cr.) Boud. & 4,8 & Esp. & $32,2 \times 16,9$ & $29,1-35 \times 14,6 \times 22,3$ \\
\hline Thelcholus crustaceus (Fuckel) Kimbrough & 9,5 & Esp. & $7,2 \times 4,7$ & $x+4,3-4,9$ \\
\hline T.stercoreus Tode ex Fr. & 19 & Frec. & $5,+\times \quad 3,1$ & x $2,9-3,4$ \\
\hline
\end{tabular}

Cain. 1969), rarios paises de Alrica (Krug \& Khan, 1989), y en İspaña (Valldoscra \& (iuarro 1992). Sus ascosporas presentan dimensiones un poco más largas que la especie tipo. pero de morfometria muy similar a las deseritas en Kenia por Krug \& Khan (1989)

$P$. miniglutinans, otra especie eonsiderada dominante, no tiene registros aparentes en Chiley su distrihueion parece guardar cierta relación con climas lluviosos. Se aisló primeramente en Mexico ( Mirza \& Cain, 1969), en Nueva Zelandia (Bell, 198.3), en Italia (Caretta et al. 1994; Caretta \& Piontelli, 1996). Sus ascosporas exceden levemente en el lago ( célula oseura), mientras el ancho se mantiene cercano a su limite inferior descrito en la literatura. Sin embargo su ascoma mestra el caracteristico peridio con pelos aglutinados y ascos con ascosporas principalmente uniseriadas. P. fimiseda (=P.fimicola, en Mir/a \& C'ain 1969), es una de las especies de amplia distribución cosmopolita ya sea en climas lirios o cálidos: generalmente se asocia a los herbivoros domestieos, especialmente bovinos (Lundqrist, 1972: Furuya \& Undagawa, 1972: Krug \& Khan, 1989, Valldoscra \& Guaro, 1992). P.fimiseda, lie registrada por Lazo encl año 1969) en Osomo, siendo citadaposteriormente 


\section{Gráfico 3. Medidas comparativas de las ascosporas de algunos Ascomycetes Patagónicos}

ESPECIES

Ascobolus immersus

Podospora argentinensis

Podospora fimiseda

Podospora miniglutinans

Podospora pleiospora

Pyxidiophora badiorostris

Pyxidiophora grovei

Thecotheus holmskjoldii

\section{ASCOSPORAS*}

Largo $\mu \mathrm{m}$

$\begin{array}{llllllllllllll}0 & 10 & 20 & 25 & 30 & 35 & 40 & 45 & 50 & 55 & 60 & 65 & 70 & 0\end{array}$

\begin{tabular}{|c|c|c|c|c|c|c|c|c|c|c|c|c|c|c|c|c|c|c|c|c|}
\hline 10 & 20 & 25 & 30 & 35 & 40 & 45 & 50 & 55 & 60 & 65 & 70 & 0 & 5 & 10 & 15 & 20 & 25 & 30 & 35 & \\
\hline 1 & & & $T$ & $T$ & $T$ & $T$ & 7 & & & 91 & 1 & & $T$ & $T$ & & & $T$ & & & \\
\hline 1 & 1 & 1 & 1 & 1 & 1 & 1 & 1 & 1 & 7 & & $\phi$ & & 1 & 1 & I & 1 & 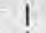 & & & \\
\hline 1 & 1 & 1 & 1 & 1 & 1 & 1 & & $=i$ & $\frac{11}{1}$ & $\stackrel{2}{T}$ & 1 & & 1 & 1 & 1 & 1 & i & & & \\
\hline T & $T$ & & 1 & 1 & 1 & $T$ & 7 & 1 & 7 & $T$ & $T$ & & $T$ & $T$ & $T$ & & $T$ & 1 & 1 & \\
\hline 1 & & & & & 1 & 1 & 1 & 1 & 1 & 1 & 1 & & 1 & 1 & & & $i$ & 1 & I & \\
\hline 1 & 1 & 1 & & 1 & 1 & $i$ & 1 & 1 & 1 & 1 & 1 & & 1 & 1 & 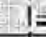 & & & 1 & I & \\
\hline 1 & $T$ & 1 & 1 & 1 & 1 & $T$ & 1 & 1 & 4 & 1 & 1 & & 1 & $T$ & 1 & 1 & $T$ & 1 & & \\
\hline 1 & i & 1 & 1 & 1 & 1 & 1 & 1 & 1 & 1 & 1 & 1 & & I & 1 & I & & & $T$ & 1 & \\
\hline 1 & 1 & 1 & 1 & & 1 & & & & & & 1 & & 1 & 1 & & & & 1 & & \\
\hline 1 & $L$ & & 1 & 1 & 1 & 1 & 1 & 1 & 1 & 1 & 1 & & 1 & 1 & $L$ & 1 & 1 & 1 & I & \\
\hline 1 & & & 1 & & 1 & 1 & 1 & & & 1 & & & 1 & 1 & & 1 & 1 & I & & \\
\hline 1 & & 1 & 1 & & 1 & 1 & I & 1 & $T$ & 1 & 1 & & 1 & 1 & & 1 & & I. & 1 & \\
\hline i & & & L & & 1 & 1 & 1 & & & & 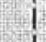 & & 1 & $i$ & & & 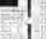 & 1 & 1 & \\
\hline 1 & & & 1 & & 2 & 1 & & & 1 & 1 & & & & I & & & 1 & 1 & & \\
\hline 1 & & & 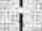 & 1 & 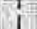 & 1 & & I. & 1 & 1 & & & 5 & . & & I & 1 & 1 & & \\
\hline & & & & & & & & & & & & & & & & & & & & \\
\hline 1 & & $i$ & 1 & & 1 & 1 & 1 & $L$ & 1 & 1 & i & & 7 & i & & 1 & 1 & i & & \\
\hline & & & 1 & & & $\frac{1}{1}$ & & 1 & 1 & 1 & I & & 7 & i & $i$ & $i$ & 1 & i & & \\
\hline I & & & & 1 & & 1 & 1 & 1 & 1 & 1 & $i$ & & i & i & & -1 & 1 & 1 & & \\
\hline 1 & & & $\perp$ & & 1 & 1 & 1 & 9 & 1 & 1 & $i$ & & I & $i$ & $\underline{1}$ & & $i$ & 1 & & \\
\hline 1 & & & & & & 1 & 1 & 1 & & 1 & 1 & & 1 & 1 & & -1 & 1 & 1 & & \\
\hline
\end{tabular}

$=$ Dimensiones especie tipo

$=$ Dimensiones especies chilenas por Lundqvist (1972), como un nuevo record para Chile. Nuevamente Lazo (1979), vuelve a aislara en lazona central. Las dimensiones (largo y ancho de la célula oscura de la ascospora), son bastante coincidentes con los registrados en la literatura, así como su pedicelo.

Dentro de las especies consideradas esporádicas, Pyxidiophora badiorostris y P.grovei tienen un particular interés en nuestro estudio, por cuanto fueron sus primeros aislamientos en Chile y al parecer no se han registrado en el hemisferio sur. Su presencia, sólo detectada alrededor de los 25 días de incubación, correspondió a una situación fortuita, debido a que sus cuellos periteciales son extremadamente dificiles de distinguir en los excrementos, confundiendose con restos vegetales (pelos). Su detección, fue posible por la agrupación gregaria de sus estructuras peritecioides y principalmente por la presencia de masas mucoides translucientes en el ápice de sus ostíolos, que contenian sus ascos y ascosporas (Hawsworth \& Webster, 1977). Inicialmente se confundieron con estructuras sinnematosas, semejante a Graphium. Solamente la preparaciónmicroscópica permitió establecer el taxon y su comparación con el trabajo de Hawksworth \& Webster (1977) y Lundqvist (1980).

Pyxidiophora (Ascomycetes, Pyxidiophoraceae), es un género particular que no forma micelio y su minuto talo peritecioide produce ascos evanescentes y ascosporas, generalmente uniseptadas transversalmente, las cuales sufren grandes variaciones morfológicas en su maduración, presentando a veces un cuerpo melaninizado apical o sub apical, que permite su dispersión por adhesión a la cutícula de varios insectos (especialmente ácaros) .

La imposibilidad de obtener subcultivos en medios artificiales y la presencia de las especies del taxon en habitat muy diversos, desde el coprófilo, los granos almacenados, los artrópodos, hasta su reconocido micoparasitismo en algunos Basidiomycota y Ascomycota (Lasiobolus, Discomycetes), ha dificultado el conocimiento de los ciclos de vida de muchas especies (Blackwell et al 1993).

La variación estructural entre las especies y sus tipos de esporas, ha llevado a una confusa e incompleta taxonomía del género (Malloch \& Blackwell, 1993). Esta situación, se aprecia también en su sistemática: desde su inclusión por Arnold (1971) ,Rogerson (1971) y Muller \& von Arx (1973), Lunqvist (1980), Barr (1990), en los Hypocreales.

Lunqvist (1980), expandió la familia, aplicando un amplio sentido genérico (sensu lato), incluyendo otros taxa como (Treleasia Speg., Mycorhynchus Sacc., Copranophilous Speg., Ascolanthanus Caill., y Acariniola Maj.). Von Arx \& van derWalt (1987), incluyelasPyxidiophoraceae en los Ophiostomatales porque 3 de sus 4 géneros poseen anamorfos en Chalara.

Blackwell \& Malloch (1989 a,b), no aceptaron esta conección Pyxidiophora-Chalara, a pesar de describir otro anamorfo sinnematoso con conidios holoblásticos y proliferaciones simpodiales o anelídicas (comunes en los Ophiostomatales), pero sus conclusiones permitieron 
posteriomente a Eriksson \& Hawksworth (1993), ubicar a Pyxidiophora en las Laboulbeniales (13lackwell, 1994).

P.badiorostris, descrita por Lundqvist (1980), en

Suecia, fue detectada posteriormente por Barraza \& Moreno (1982), en España. Las caracteristicas morfológicas de csta especie concuerdan con la de la literatura. P.grovei ha sido registrada en Inglaterra y Suecia (Hawksworth \& Webster, 1977: Lundqvist, 1980). La determinación en nuestromaterial ha sido problemática, por cuanto tuvimos diferencias en el largo de las ascosporas, con un premedio menor que el descrito para la especie tipo (Tabla 1). Sin embargo las células del cuello del perítecio y las de la pared peritecial coinciden, no asi lasmedidas de suascoma (5)-6() de diam. en la base, con cuellos de 120-130 de largo y 25-28 de ancho en la base). Por las dimensiones del ascoma y el largo de las ascosporas, semeja a P. petchii (Breton \& Faurel) Lundqv., pero ditiere en el ancho de las células de la pared peritecial $y$ en el ancho de las ascosporas y por ser una especie caulicola y no coprólila. L a presencia escasa e inmadura de su anamorfo en Chalara, fue otra razón que nos inclinaron a considerarla como P.grovei.

Como P.hadiorostris y P.grovei, son de habitat coprólilo, la escasa detección en nuestro hemislerio es atribuible posiblemente a su dilicil visualización $y$ a los pocos estudios ecológicos relacionados con este ambiente füngico.

Las 2 especies de Thecotheus ( $T$. pelletieri $y T$. holmskjoldii), lueron de erecimiento tardio $y$ aisladas al linal del tiempo de incubación de las placas, lo que pudo condicionar cierto estress hidrico que no permitió a ambos taxa un rango de madurez completa, por lo que sus apotecios mantuvicron un color blanco cremoso y no presentaron tonalidades café a púrpura. Sus escasas presencias, también inlluyeron en la posibilidad de un mejor análisis de éstas, asi como los negalivos subeultivos en agar estiercol. Esta situación se describe en la líteratura (Conway 1975), considerando especialmente a T.pelletieri como un organismo tardio en su fructilicación y sucesión. siendo por esta causa poco lrecuente su visualización en los excrementos.

En general la micromorfología de T.pelletieri, coincide bien con la descripción de la especies (Kimbrough, 1969): sus típicos ascos de 32 esporas ), su excipulum ectal con hilas superliciales bulbosas, sus ascos que se tiñen enteros de azul con el reactivo de Melzer y sus ascosporas jóvenes de paredes gruesas $( \pm 3 \mu m)$ y lisas, dispuestas irregularmente en el asco, sinuna apiculo polar. Sin embargo el largo y ancho de sus ascosporas presentó medidas que difieren del original $(29,1-35 \times 14,6-22,3(\bar{x}=32,2 \times 16,9)$ especialmente en el ancho: La disminución del tamaño de las esporas se ha observado principalmente en climas cálidos (Krug \& Khan, 1987 y Redhead \& Gimns, 1980), para algunas Sordariaceae y Basidiomyectes, situación también descrita en la India para la misma especie (Kaushal , 1980) que presentó ascosporas más pequeñas $(28-32 \times 14-17)$. Sin embargo, las condiciones climáticas de nuestra región patagónica son totalmente distintas. Esto puede deherse a la lalta de madurcz del ascomata orepresentar a un ecotipo distinto bajo condiciones climáticas adversas.

Lasegunda especiedel género, muestra gran similitud con la anterior en cuanto a las caracteristicas del apotecio $y$ de sus parálisis infladas, pero la presencia deamplios ascos octosporados con un marcado operculo, esporas apiculadas con paredes de 2 capas, la separa rápidamente de $T$. pelletieri, sin embargo también se obtuvieron medidas más reducidas de sus ascosporas

Analizando la clave de Krug \& Khan (1987), no existe una semejanza con ofras especies octosporadas, por lo tanto se consideró morfologicamente similar a T.holmskjoldii (=Ascophamus holmskjoldii). Kimbrough (1969), sinonimiza a esta especic con $T$. cinereus, pero muchos autores prelieren mantenerla separada; aunque presenta también asessporas apiculadas polarmente, su episporio es caracteristico por sus finos gránulos que aumentan de tamaño en los polos (Van Brumelen, 1967; Ekblad, 1968; Dennis 1978; Korf \& Zhuang, 1991; ). T. harasinus, es otra especie similar a T. pelletieri y T.holmskjoldii, sin embargo sus ascosporas a pesar de presentar apículos similares tienen un episporio con gruesos gránulos y sus ascospo-

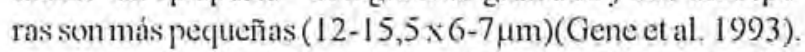

T. cinereus fue aislado en la zona central de Chile por Lazo (1979), situación que podria extrapolarse también a una mayor distribución de esta especie en el territorio nacional.

A pesar que una buena parte de los taxa descritos en esta localidad austral como en otras zonas del pais, se han reportado en excrementos de bovinos y equinos en climas muy diversos. Nuestros registros solo reflejan una parte de la micota coprófila presente en una época estacional, que debe asociarse al tipo de clima reinante, al tipo de animal y a los pastos, situación que junto a variados factores bióticos y abióticos afecta en gran medidar la diversidad de especies en la comunidad. Estimabamos una mayor riqueza y densidad de especies, llamandonos la atención la falta de otros Aseomycetes comunes en estos sustratos (Chatomium, Sordaria, Kernia ,etc), así como la escasa cantidad de Hyphomycetes; en especial los simnematosos (totalmente ausentes).

A pesar de que nuestro sustrato representa solo un pequeño universo, constituyc un ecosistema con una alta biodiversidad lüngica $y$ por ende una comunidad cuya estabilidad debicra cstudiarse en el tiempo, como aporte de información ecológica. 


\section{REFERENCIAS}

Arx,J.A. von \& Van der Walt,J.P. (1987). Ophiostomatales and Endomycetales. In: G.S. de Hoog, M.; Smith, M. Th. \& Weijmann, A.C.M. (Eds). The expanding realm of yoast-like fungi. Stud. Mycol, 30:167-176

Arnold, G.R.W. (1971), Ober eininge neue Taxa und Kombinationen der Sphaeriales. Z. Pilzk, 37:187-198

Barraza, J.M. \& Moreno,G. (1982). Pyxidiophora hadiorostris Lundq. y Pyxidiophora fimbriata sp. nov., en España (Pyrenomycetes). Cryptogamia, Micol. 3:41-48

Barr, M.E. (1990). Prodomus to nonlichenized, pyrenonycetous members of Class Hymenoascomyceles: Nycolaxen 39:43-184

Bell, A. (1983). Dung fungi. an illustrated guide to coprophilous fungi in New Zeland. Vicloria University Press. Wellington, N.Z.

Bills, F.G. (1995).Analyses of microfungal diversity from a user's perspective. Can.J.Bot.73(Suppl.1):S33-S41

Blackwell, M. (1994). Minute mycological mysteries: The intluence of arthropods on the lives of fungi. Mycologia 86:1-17

Blackwell,M. \& Malloch,D, (1989a). PyxidiophoratA link between The Laboulbeniales and hyphal ascomycete Mem.N.Y.Bot. Gard. 49:23-32

Blackwell,M. \& Malloch,D. (1989b). Pyxidiophora: Life history and arthropod associations of two species. Can. J. Bot. 67:25522562

Blackwell,N; Spatafora,J.W; Malloch,D \& Taylos,J,W. (1993). Consideration of higher taxonomic ralationships involving Pyilliophora. In: Wingfield.J; Seifert,A.K, Webber,F.J (Eds.) Ceratocystis and Ophiostoma, Taxonomy, Ecology and Pathogenicity. APS Press, St. Paul, Minnesota, pp. 105-108

Brummelen, J.van. (1967). 'A world monograph of the genera Ascobolus and Saccoholus (Ascomycetes. Pezizales). Persoonia suppl, vol. 1 Rijks-Herbarium, Leiden. The Nederlands.

Caretta,G.; Mangiarotti,A.M. \& Piontelli, E. (1994). Coprophilous fungi on horse goat and sheep dung from Lombardia (ltaly), Mic. Ital. 2:11-20

\& Piontelli ,E. (1996). Coprophilous fungi from confined deers in Pavia (Lombardia,Italy). Boletín Micológico $11: 41-50$

Conway, K.E. (1975). Ascocarp Ontogeny and imperfect state of Thecotheus (Pezizales, Ascomycetes) Mycologia 67:241-252

Dennis, R.W.G. (1978). British Ascomycetes. J.Cramer, Vaduz

Eriksson,O.E. \& IIawksworth,D.L. (1993). Index to notes 9691529 Syst. Ascomycetum 9:1-38

Eckbad F.E. (1968). The genera of Opcrculate Discomycetes, A Revaluation of their taxonomy, Phylogeny and nomenclature. Nytt. Mag. Bot. 15: 1-191

Furuya,K. \& Udagawa,S-I. (1972). Coprophilous Pyrenomyce- les from Japan.I. J. Gen Appl. Microbiol. 18: 433-454

Gene,J.; EIShafie,A.E.\& Guarro J. (1993). Two new coprophilous Pezizales from the sultanate of Oman. Mycotaxon 46:275-284

Hawkworth,L.D.\& Webster,J.(1977). Studies on Mycorhynchus in Britain. Trans. Br. mycol Soc. 68:329-340

Kaushal, S.C. (1980)). A new species of Thecothens (Pezizae, Pezizales) liom western Himalayas. Bot. Notiser 133:319-321

Kimbrough,J.W. (1969). North American species of Thecotheus (Pezizene, Pezizaceac). Mycologia 61:99-114

Korf, R.P. \& Zhuang Wen-Ying. (1991). A preliminary Doscomycete llora of Macaronesia: Part 12. Pyrenomatineae, and, Pezizincae, Ascobolaceae Mycotaxon 40:307-318

Krug ,J.C. \& Khan, R.S.(1989). A new species of 7hecotheus from East Asia. Mycologia 79: 200-203

Lazo, W. (1979). Honges coprólilos de Chile, Archivos de Biol. y Med. Exper, 12:637 (Resumen)

Lundqvist, N. (1972).Nordic Sordariaceae S.Lat. Symb.Bot. Upsal. 20:1-374

Lundqvist, N. (1980). On the genus Pyxilliophora sensu lato (Pyrenomycetes).Botanisk Notiser 133:121-144

Malloch,D.\& Blackwell,M. (1993). Dispersal biology of the ophiostomatoid fungi. In: Wingfield,J; Seifert, A.K, Webber,F.J (Eds.) Ceratocystis and Ophiostoma, Taxonomy, Ecology and Pathogenicity. APS Press, St. Paul, Minnesota, pp.195-206

Montaldo,P, (1976). Análisis ecológico de mallines de Aisén. Agro Sur $4: 106-110$

Miller,L.S.(1995). Functional diversity in fungi. Can. J. Bot, 73(Suppl.1): S50-S57

Mirza, J.II. \& Cain, R.F. (1969). Revision of the genus Podospora. Can.J.Bol. 47:1999-2048

Müller, E.\& von Arx,J.A.(1973). Pyrenomycetes: Meliolales, Coromophorales, Sphaeriales. In : Ainswvorth, G.C; Sparrow,F.K.\& Sussman,A.S. eds, The fingi: an advances treatise. Vol 4a. N.Y., London, Academic Press. pp.87-132

Muroi,T.\& Udagawa,S.(1984). Some coprophilous Ascomycetes from Chile. In: H.Inoue, Ed. Studies on Cryplogams in Southem Chile pp.161-167

Piontelli, E;Toro, M.A \& Caretta,G.(1981). Coprophilous fungi of the horse. Mycopath. 74:89-105

Quintanilla, V.(1983). Geografia de Chile. Tomo III. Biogeografia,.Instiluto Geográfico Militar. Santiago

Redhead,S.A. \& Ginns,I. (1980). Cyptotrama asprata (Agaricales) from North America and notes on the five other species of Cyptotrama sect. Xerulina. Can. J. Bot, 58:731-740 
Rogerson,C.T. (1971). The Hypocrealean fungi (Ascomycetes, Hypocreales) Mycologia 62: 865-910

Shearer,A.C.(1995). Fungal competition. Can.J.Bot. 73 (Suppl.1): S1259-S1264

Spegazzini,C.(1912). Anal. Mus. Nac. Buenos Aires 23:49

(1921).Mycetes Chilenses. Bol. Acad. Nac. de Cienc.

Córdoba 25:1-124

Udagawa,S.(1980). Some new or noteworthy coprophilous Pyrenomycetes from South America. Trans.Mycol. Soc.Jap. 21:283298

Valldosera,M. \& Guarro,J.(1988). Some coprophilous Ascomycetes from Chile. Trans Br. mycol. Soc. 90:601-605
\& Guarro,J.(1992). Estudios sobre hongos coprófilos aislados en España.XVIII. Recopilación de la bibliografía existente y relación de todos los Ascomycetes citados. Bol. Soc. Micol. Madrid 17: $39-55$

Wicklow, D.T; Angel,K,\& Lussenhop, J. (1980). Fungal community expression in lagomorph versus ruminant feces. Mycologia 72:1015-1021

Yocom, P. \& Wicklow,D.T.(1980).Some soil and coprophilous fungi from the south Pacific area. Mycologia 52:552-556 

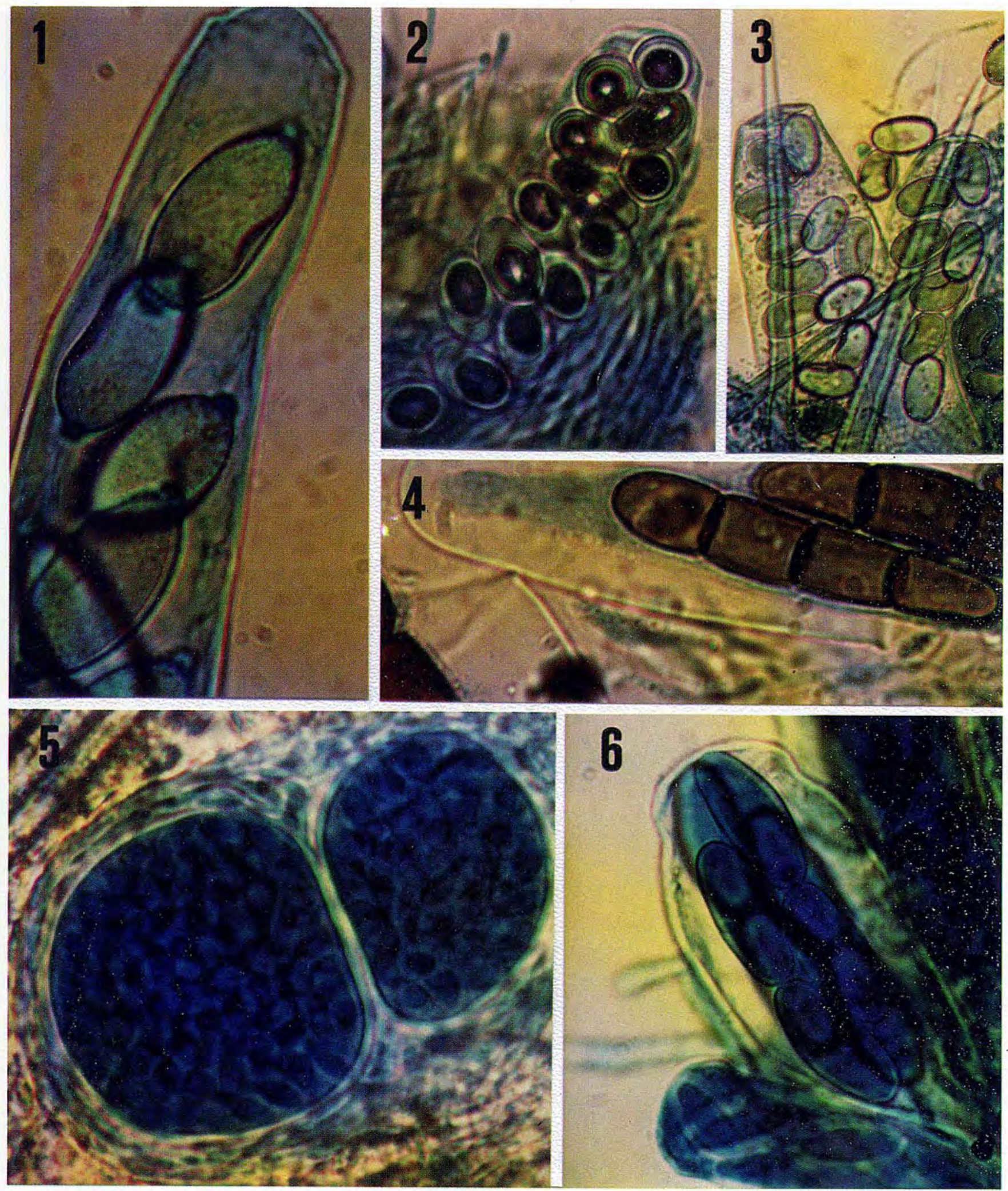

1.- Thecotheus holmskjoldii, asco y ascosporas apiculadas 1000x. 2.- Coprotus duplex, ascos y ascosporas 1000x. 3.Lasiobolus intermedius, ascoma, ascos y ascosporas 1000 x. 4.- Sporormiella intermedia, asco y ascosporas 1000x. 5.Thelebolus stercoreus, ascoma y 2 ascos multiesporados 1000x. 6.- Saccó̈oius círinus, ascos y ascosporas 1000x. 

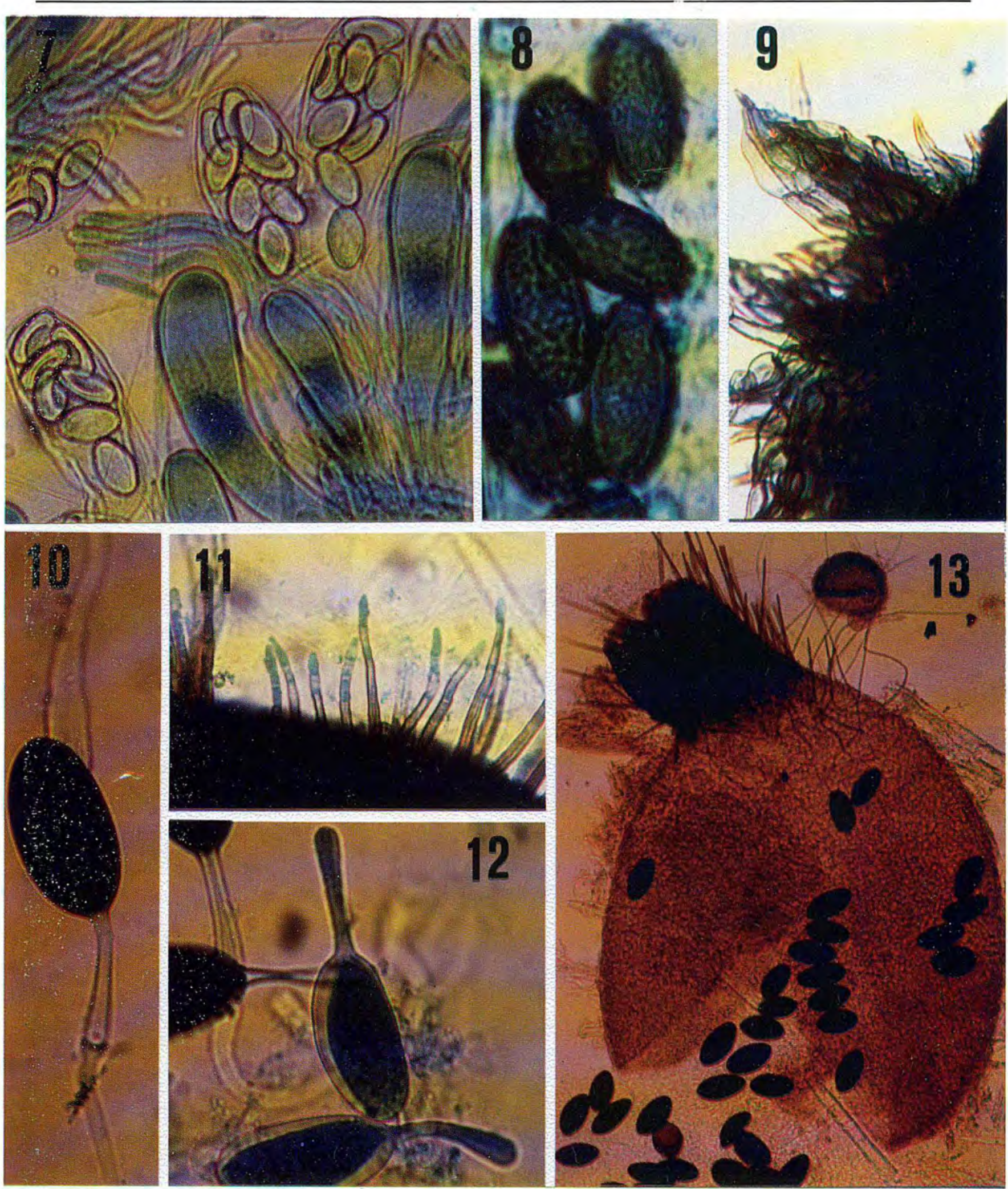

7.- Iodophanus carneus, paráfisis, ascos y ascosporas 400x. 8.- Ascobolus perplexans, ascosporas 1000x. 9. Podospora conica, pelos aglutinados en el cuello 400x. 10-11-12 - Podospora fimiseda. 10.- Ascosporas y sus apéndices 1000x. 11.Pelos periteciales 1000x. 12.- Ascosporas jóvenes y apéndices 1000x. 13-14.- Podospora ostlingospora. 13.-Ascoma y ascosporas 200x. 

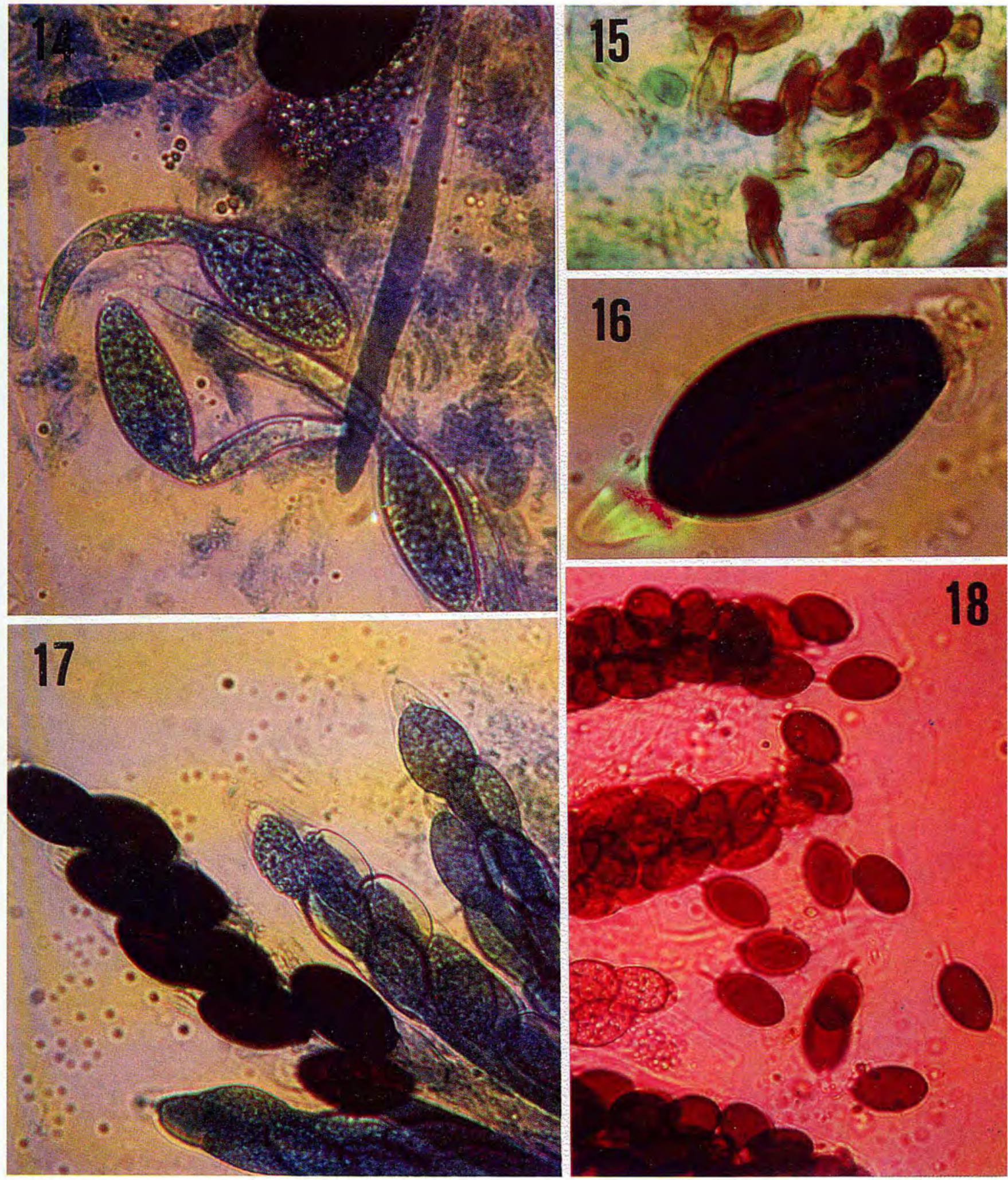

14.- Ascosporas jóvenes con apéndices basales 1000x. 15-16-17.- Podospora argentinensis. 15.- Pelos tubulares del cuello del peritecio 1000 x. 16.-Ascospora y sus apéndices 1000 x. 17.- Ascos con ascosporas maduras y jóvenes. 18.Arnium leporinum, ascospora con sus apéndices 400x . 


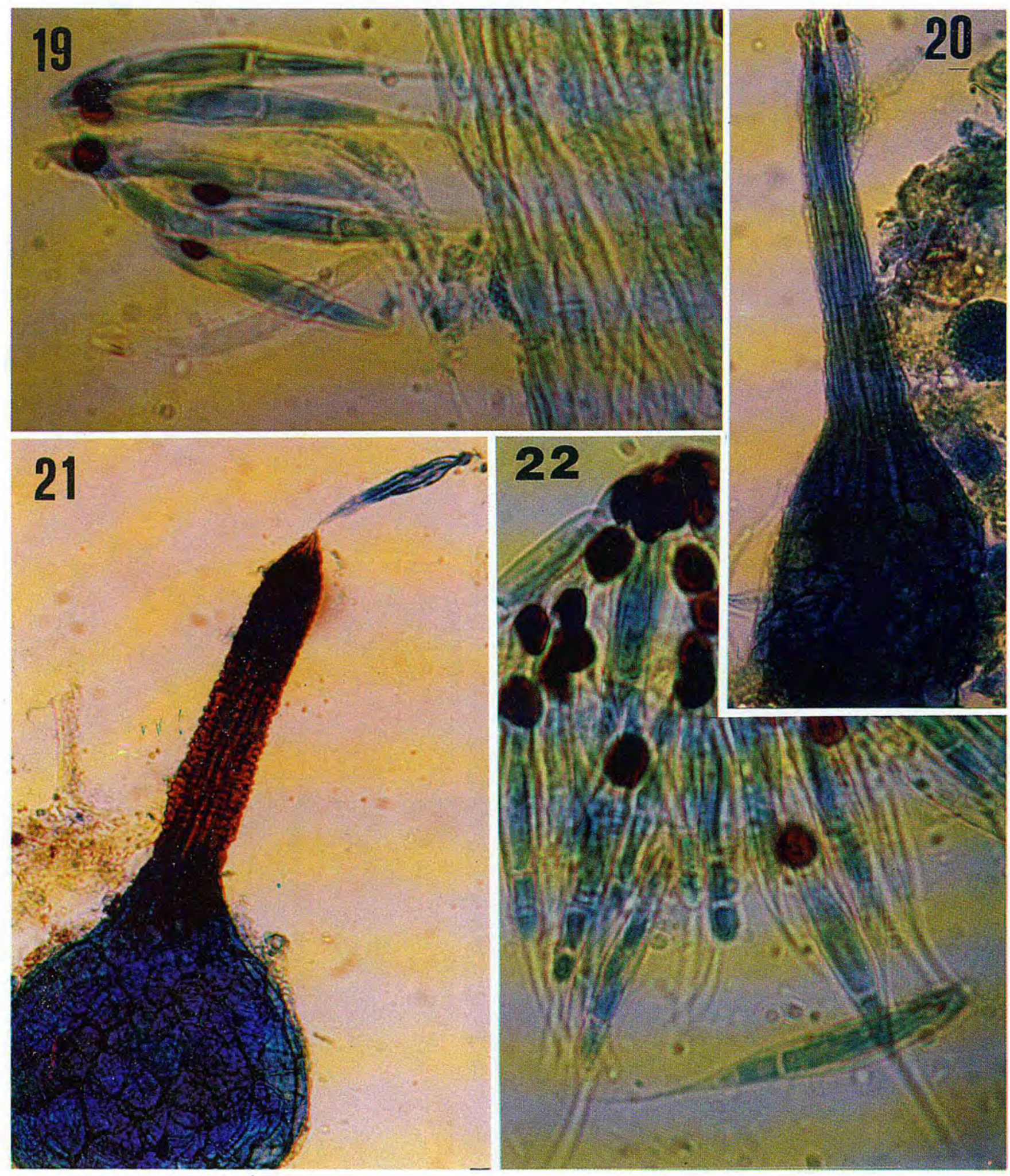

19-20.- Pyxidiophora grovei. 19.-Ascosporasy parte del cuelloperitecial 1000x. 20.-Ascoma 400x. 21-22.- Pyxidiophora badiorostris. 21.- Ascoma y ascosporas saliendo del ostiolo 400x. 22.- Ascosporas 1000x. 